\title{
Serum Homocysteine and the Short-Term Outcome of Ischemic Stroke
}

\author{
Alia Saberi ${ }^{\mathrm{a}}$, Mersedeh Javadzadeh Saber ${ }^{\mathrm{a}}$, Seyed Ali Roudbary ${ }^{\mathrm{a}, \mathrm{b}}$, \\ Arezoo Shirzani ${ }^{\mathrm{a}}$
}

\begin{abstract}
Background: Homocysteine is considered as one of the factors affecting the prognosis of ischemic stroke. The aim of this study is to determine the relationship between homocysteine level and ischemic stroke outcome.
\end{abstract}

Method: This cross-sectional study was conducted between December 2010 and September 2011 on 145 patients with ischemic stroke in middle cerebral artery (MCA) territory, after approval by Guilan University of Medical Science's Ethics Committee. Patients with a history of stroke, brain lesion, liver or kidney disorders, pneumonia, sepsis and who had seizure at the beginning or during hospitalization were excluded. Uncontrolled diabetes, anemia and dramatic blood pressure reduction in admission were defined as confounding factors. In the first week, serum level of homocysteine was measured and the functional independence and status of patients were measured with functional independence measure (FIM) and Barthel index (BI) in the first week and first month after stroke. Data were analyzed by t-test, ANOVA, Mann-Whitney U, KruskalWallis tests and backward stepwise logistic and linear regression analysis using SPSS 19.

Results: One hundred and forty-five patients $(55.9 \%$ men and $44.1 \%$ women) with mean age of $68.3 \pm 13.8$ years old were evaluated. Serum level of homocysteine was determined as an independent predictor for mortality in the first week $(\mathrm{P}=0.017$; OR $=$ 1.179; $95 \% \mathrm{CI}: 1.03$ - 1.35). No significant correlation was found between serum homocysteine level and BI and FIM in the period of study $(\mathrm{P}>0.05)$.

Conclusions: Homocysteine decreases mortality rate in patients with stroke in the first week but does not affect the functional outcome of alive patients.

Manuscript accepted for publication February 5, 2014

${ }^{a}$ Neurology Department, Guilan University of Medical Sciences, Rasht, Guilan, Iran

${ }^{\mathrm{b}}$ Corresponding author: Seyed Ali Roudbary, Neurology Department, Guilan University of Medical Sciences, Rasht, Guilan, Iran.

Email: roudbary@gums.ac.ir

doi: http://dx.doi.org/10.14740/jnr265w
Keywords: Homocysteine; Outcome; Stroke; Ischemic stroke

\section{Introduction}

Stroke is one of the most disabling disorders in humans. All types of ischemic stroke are caused by embolic or thrombotic occlusion of one of the feeding arteries of brain. As the perfusion to the area of occluded artery is stopped, a cascade of molecular and cellular events starts and continues that leads to cell death. As more brain cells die, disability will be more. Multiple factors are effective on progress and intensity of events that result in different outcomes of patients with stroke in the territory of the same occluded arteries.

Homocysteine is a known and approved risk factor for stroke and atherosclerosis, especially in the young adults [1-4]. Homocysteine is an amino acid containing sulfydril and needs vitamin B6, B12, folic acid and betaine for the metabolism [3]. Nutritional and genetic factors play a role in homocysteine metabolism disturbance [5, 6]. Homocysteine involves several pathophysiologic pathways, including damage to wall of arteries and interfering with the coagulation system. This amino acid has a pre-oxidative and pre-inflammatory effect and induces the progression of vasoconstriction, disturbance of structure and function of endothelium and atherosclerosis. It also acts as a pre-thrombotic factor with increasing coagulability and platelets aggregation. A significant relationship was discovered between serum level of lipoprotein and homocysteine and high serum level of homocysteine regulates the lipoproteinemia toxicity in ischemic stroke $[1,2,5,7,8]$. Some disorders increase homocysteine serum level, including chronic renal failure, hypothyroidism, pernicious anemia, cancers of breast, ovary and pancreas, acute lymphoblastic leukemia and some drugs such as methotrexate, phenytoin and theophylline and smoking [9].

In recent years, homocysteine is under investigation and research as a factor affecting the prognosis of stroke, but their results are in controversy [1, 10-14]. It has been found that homocysteine induces oxidative stress in vitro and hy- 
Table 1. Comparison of the Functional Independence of Ischemic Stroke Patients Based on Serum Homocysteine Status

FIM in first month

Total

$\begin{array}{lll}\text { Dead } & \text { Low } & \text { Medium }\end{array}$

$\begin{array}{lllll}\begin{array}{l}\text { Serum homocysteine } \\ \text { Normal }\end{array} & 44(41.9 \%) & 29(27.6 \%) & 32(30.5 \%) & 105(100.0 \%) \\ \text { High } & 24(60.0 \%) & 4(10.0 \%) & 12(30.0 \%) & 40(100.0 \%) \\ \text { Total } & 68(46.9 \%) & 33(22.8 \%) & 44(30.3 \%) & 145(100.0 \%)\end{array}$

$P=0.05$.

perhomocysteinemia reduces ATPase's activity [11].

In this study, we evaluated the serum level of homocysteine in patients with ischemic stroke and its relationship with patients' functional statue and independence in order to if there was any relationship; we could prescribe medications that affect the metabolism and serum level of homocysteine in acute and post-acute phase of stroke to improve its outcome.

\section{Materials and Methods}

This cross-sectional study was conducted between December 2010 and September 2011 on 145 patients with ischemic stroke in MCA territory after approval by Guilan University of Medical Science's Ethics Committee. Patients with a history of stroke, previous brain lesion, liver or kidney disorders, pneumonia, sepsis and who were having a seizure at the beginning or during hospitalization were excluded. Uncontrolled diabetes, anemia and dramatic blood pressure reduction in admission were defined as confounding factors. After fulfilling the informed consent by patients or their legal responders, in the first 7 days after stroke, fasting blood samples (after $8-10 \mathrm{~h}$ of fasting) were taken from all patients to measure serum level of homocysteine. The samples were spilled in tubes containing ethylenediaminetetraacetic acid and centrifuged immediately. The resulting plasma is kept in $-20{ }^{\circ} \mathrm{C}$ and the serum levels of homocysteine were measured by high-performance liquid chromatography technique with fluorescence recording. Normal level of serum homocysteine was considered as $15 \mu \mathrm{mol} / \mathrm{L}$ and the values higher than this were considered as high homocysteine level or hyperhomocysteinemia.

Patients were examined at the end of the first week and first month. The patients' functional independence was measured by functional independence measure (FIM) score and patients were divided into three categories: low score (FIM $\leq 40)$, intermediate score $(41 \leq \mathrm{FIM} \leq 80)$ and high score (FIM $>80$ ). Furthermore, the changes of FIM score were determined in above-mentioned duration. As well, the patients' functional status was measured by Barthel index (BI) and also the patients were divided into three categories: bad prognosis $(\mathrm{BI}<40)$, intermediate prognosis $(41 \leq \mathrm{BI} \leq 70)$ and good prognosis $(\mathrm{BI}>70)$. Also the patients' mortality was recorded in the study period.

All information of patients was entered in a previous designed heck list. For data analysis, the parametric tests such as t-test and ANOVA, and nonparametric tests such as MannWhitney U and Kruskal-Wallis test were used in SPSS 19.

\section{Result}

A total of 145 patients (55.9\% men and the others women) with ischemic stroke in MCA territory were enrolled in present study. Their mean age was $68.3 \pm 13.8$ years old and the mean of their serum level of homocysteine was $12.99 \pm 5.6$ $\mu \mathrm{mol} / \mathrm{L}$. The serum level of homocysteine was high among $27.56 \%$ of them (40 patients).

Evaluation of functional independence in the first week (based on FIM) showed that all alive patients had low FIM score and none of them had intermediate and high score. Therefore, comparing the state of various levels of homocysteine was not feasible according to FIM.

Evaluation of functional independence in first month (based on FIM) showed that none of the alive patients had high FIM score and severity of FIM established a near significant statistical relationship with serum homocysteine status in the first month (Table 1).

Assessment of functional status (based on BI) in the first week showed that all alive patients had intermediate BI and none of them had low or high BI. Also assessment of func- 


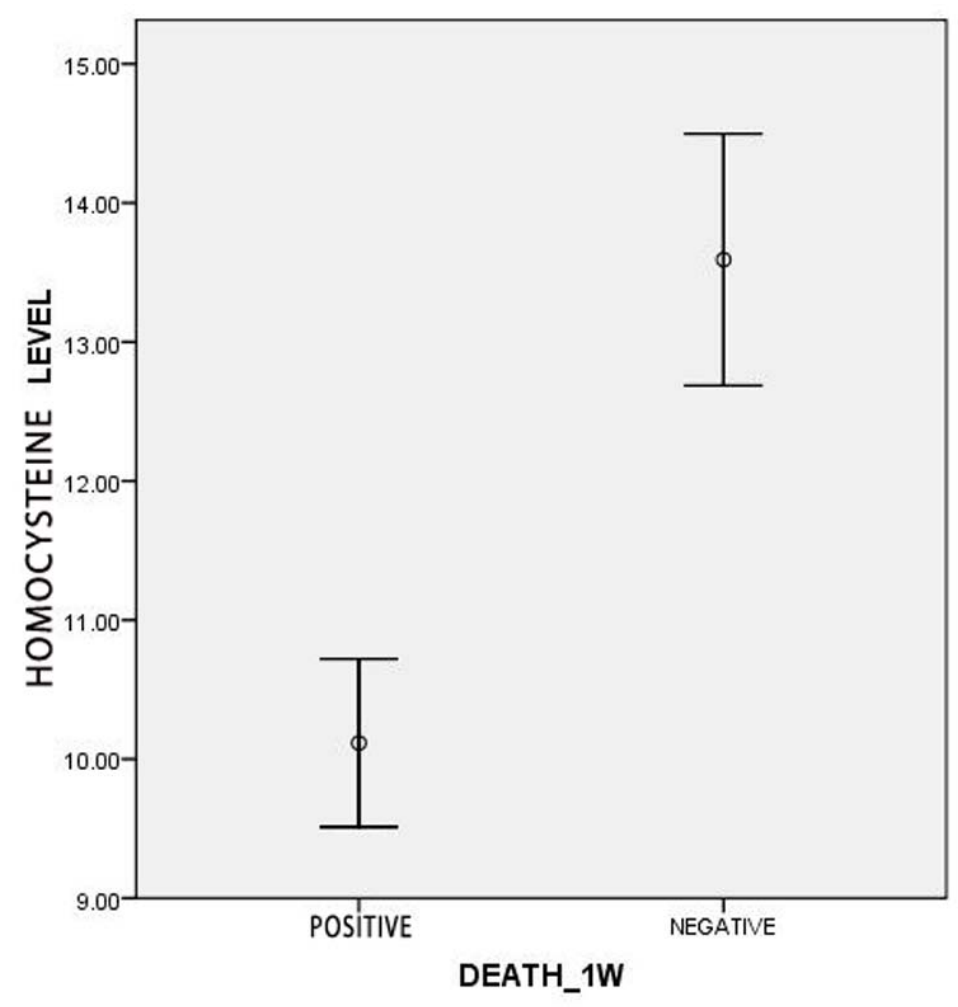

Figure 1. Comparison of the serum level of homocysteine among dead and alive patients in the first week.

tional status (based on $\mathrm{BI}$ ) in the first month showed that all alive patients had high BI and none of them had low or intermediate BI. Therefore, comparing the status of various levels of homocysteine was not feasible according to BI in both first week and first month.

Evaluating of mortality, FIM and BI of the first week and the first month according to two quantitative variables, age and serum level of homocysteine, showed that the mean age of the alive and dead patients had significant differences. So that in the first week evaluation, the mean ages of dead patients and alive patients were $58.44 \pm 15.23$ and $70.02 \pm$ 11.71 years old $(\mathrm{P}=0.001)$. As well the mean ages of patients at the end of the first month were $64.29 \pm 12.05$ years old in alive patients and $70.82 \pm 13.21$ years old in dead patients $(\mathrm{P}=0.001)$. Also the mean of homocysteine level was $10.12 \pm 1.84 \mu \mathrm{mol} / \mathrm{L}$ in dead patients and $13.59 \pm 6.03$ $\mu \mathrm{mol} / \mathrm{L}$ in alive patients with significant difference during the first week $(P=0.014)$, but there was not any significant difference in the first month: $12.44 \pm 5.22 \mu \mathrm{mol} / \mathrm{L}$ in dead subject vs. $13.73 \pm 6.23 \mu \mathrm{mol} / \mathrm{L}$ in alive patients $(\mathrm{P}=0.115)$ (Fig. 1, 2).

Also the first week mortality rate among patients with normal homocysteine was $22.9 \%$ (24 patients) and in hyper- homocysteinemic patients was $2.5 \%$ (one patient) with statistically significant difference $(P=0.004)$, but there was not any significant difference in the first month (0.0277).

Prediction area of mortality during the first week based on homocysteine levels by receiver operating characteristic (ROC) curve (area under the curve (AUC)) was $0.656 \pm$ 0.047 , which is statistically significant $(\mathrm{P}=0.014 ; 95 \% \mathrm{CI}$ : $0.564-0.748$ ) (Fig. 3).

Also in multivariant analysis, the serum level of homocysteine remained in the model and was determined as a predictor of mortality in the first week after ischemic stroke. So that, by increasing $1 \mu \mathrm{mol} / \mathrm{L}$ of serum level of homocysteine the first week mortality probability decreases 1.179 times (P $=0.017 ; \mathrm{OR}=1.179 ; 95 \% \mathrm{CI}: 1.031-1.350$ ).

Prediction area of mortality during the first month based on homocysteine levels by ROC curve (AUC) was $0.577 \pm$ 0.048 ( $\mathrm{P}=0.115$; 95\% CI: $0.482-0.671)$, of first week FIM was $0.569 \pm 0.81(\mathrm{P}=0.379$; 95\% CI: $0.411-0.728)$, of first month FIM above 40, $0.567 \pm 0.048(\mathrm{P}=0.167 ; 95 \%$ CI: $0.473-0.661)$ and above $80,0.056 \pm 0.521(\mathrm{P}=0.689$; $95 \%$ CI: $0.412-0.630$ ), of first week BI was $0.569 \pm 0.86$ (P $=0.395 ; 95 \%$ CI: $0.401-0.738)$ and of first month BI was $0.567 \pm 0.048(\mathrm{P}=0.167 ; 95 \% \mathrm{CI}: 0.473-0.661)$, none of 


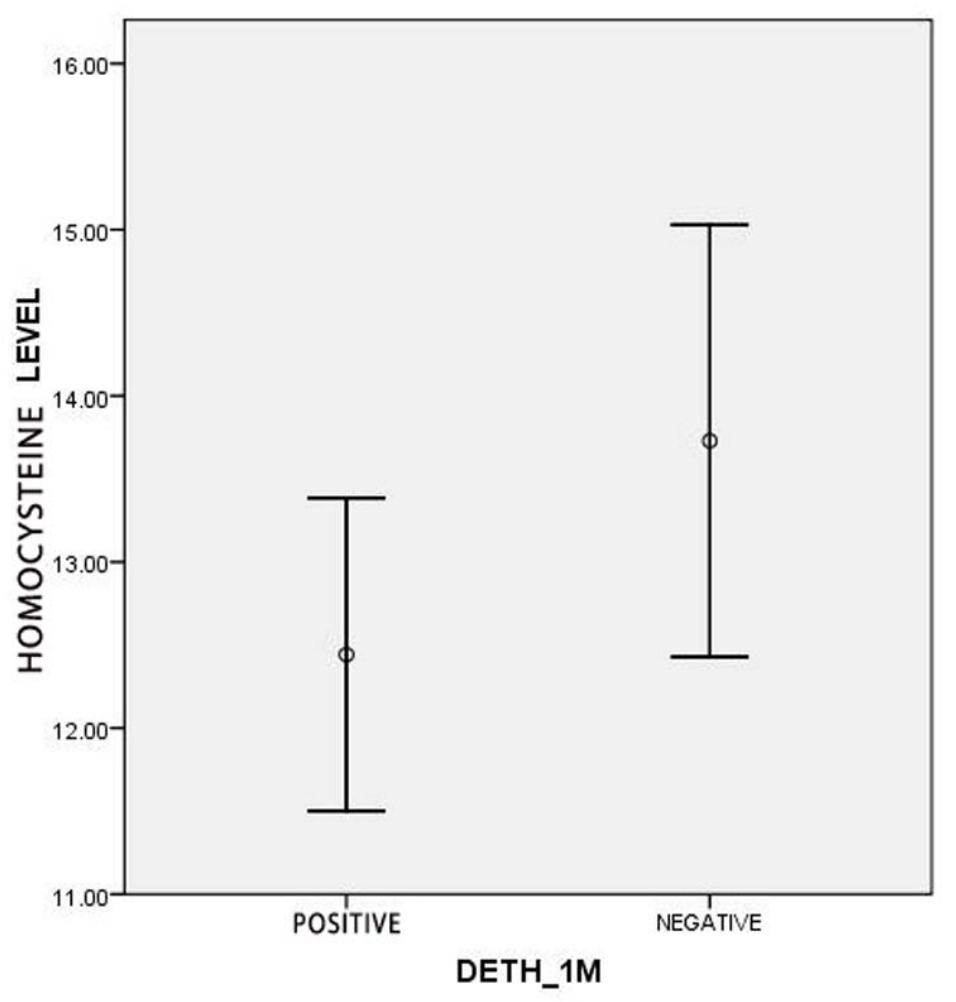

Figure 2. Comparison of the serum level of homocysteine among dead and alive patients in the first month.

which was not statistically significant.

Regression model of predictive factors of FIM and BI in the first week and the first month did not show a significant relationship with confounding factors.

Since serum level of homocysteine is the only predictive factor of mortality in the first week, its cut-point for predicting first week mortality was determined as $10.1 \mu \mathrm{mol} / \mathrm{L}$ with $66.7 \%$ sensitivity and $64 \%$ specificity.

\section{Discussion}

In the present study, the serum level of homocysteine more than $15 \mu \mathrm{mol} / \mathrm{L}$ was defined as hyperhomocysteinemia and the mean of serum level of homocysteine of all patients was less than this point $(12.99 \pm 5.6 \mu \mathrm{mol} / \mathrm{L})$ and only $27.6 \%$ of them (40 of 145 patients) were hyperhomocysteinemic.

By folic acid and vitamin B12 insufficiency, which are essential for homocysteine metabolism, the serum level of homocysteine will be increased. In Middle East area such as Iran, especially among people involved by stroke, maybe vegetable consumption is less common than in East Asia and Indian populations that most of them are vegetarian. Thus it is suspected that vitamin B12 and folic acid deficiency is less frequent in our population and homocysteine levels rise less than those populations. So frequency of the hypercysteinemia in our study is less than a study conducted by Kalita in India $(66.6 \%)$ [5]. In addition, genetic factors affect the level of homocysteine, so that the prevalence of polymorphism and mutation of methylentetrahydrofolate reductase varies in different communities $[3,15,16]$.

In some studies, homocysteine is considered as a cause of large vessel stroke [17]. Whereas in another study, homocysteine is associated with small vessel stroke [18] and in the others there is no difference in the size of stroke [7]. Since one of the main factors that affects the performance of stroke patients is the location and size of infarct, so for the unification of the patients, all patients included in this study were with ischemic stroke in the right and left MCA supply area while the other studies did not evaluate specific artery territory [13] and this was a confounding effect on their results. Patients with hyperhomocysteinemia were not matched for age, sex and other risk factors of stroke, but the effects of these confounding factors in multivariate analysis were omitted.

In this study, there was no significant relationship between serum level of homocysteine and means of FIM and $\mathrm{BI}$ in first week and first month. But in first month, BI in 


\section{ROC Curve}

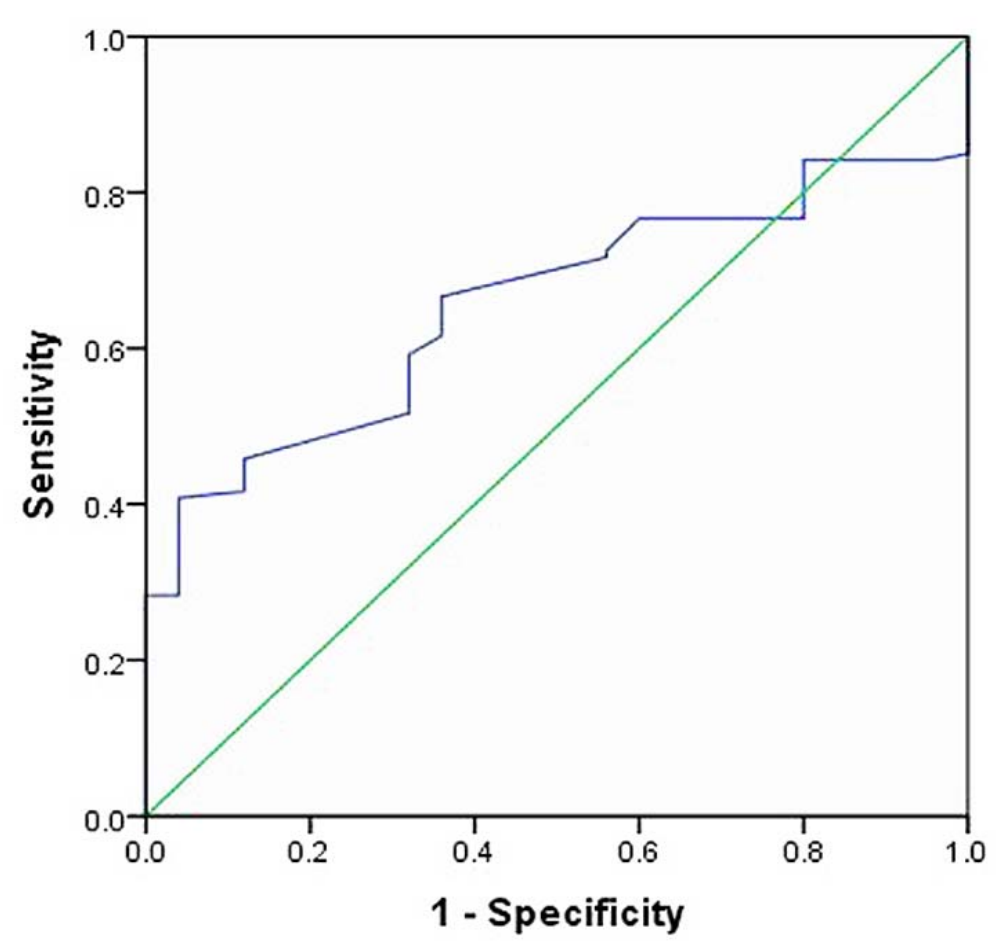

Diagonal segments are produced by ties.

Figure 3. ROC curve of the predictive role of homocysteine in first week mortality.

patients with normal level of serum homocysteine was more than hyperhomocysteinemic patients $(\mathrm{P}=0.07)$, so that the percentage of patients with BI more than 70 in normal homocysteine patients $(58.1 \%)$ was more than patients with high homocysteine levels $(40 \%)$. But since all alive patients had high BI, this is only a comparison between live and dead patients and does not show association between serum level of homocysteine and the amount of BI.

There was no significant association between FIM score and serum level of homocysteine in the first week, but there was a significant association between homocysteine status and FIM scoring of the first month $(\mathrm{P}=0.05)$. In other words, patients with normal homocysteine level had higher FIM score of first month than patients with higher homocysteine level but this association was not with absolute value of homocysteine and disappears after adjusting for confounding factors.

Although most studies agree with the effect of hypercys- teinemia on the incidence of stroke, few studies have evaluated its effect on stroke outcomes and they are not compatible with each other also.

In one study by Howard et al in 2000 among 76 patients involved by ischemic stroke, serum level of homocysteine in two groups with Rankin score above 2 and below 2 did not differ [17] and it seems that it is congruent with our results. Of course there are some methodological differences so that we compared the function of normal homocysteine and hypercysteinemic groups with each other wherever Howard compared the serum homocysteine level of two groups with different function scores. Also the above-mentioned study used Rankin score that evaluates patient's disability, wherever we assessed the functional outcome by FIM and BI.

In the other study that has been implemented in 2004 by Mizrahi et al, fasting serum level of homocysteine was measured in 113 patients with acute ischemic stroke during 2 - 7 days after the ischemic attack. The patients were divided 
to two groups: normal homocysteine and hyperhomocysteinemic, exactly similar to our study. Then patients' functional independence was evaluated at admission and discharge. They were divided into three groups based on FIM score (low $<40,41<$ intermediate $<80$ and high $>80$ ). Finally, it has been determined that there is no association between serum level of homocysteine and functional outcome of patients after rehabilitation of stroke. In this study, patients who were hospitalized for less than 7 days were excluded and it may be a confounding factor [14]. In spite of this difference and enrolling of all patients with stroke in all arterial territories, its results are matched with ours, considering the similarity of scores which are used and their methodologies.

Another study carried out in 2007 in Nigeria by Okubadejo showed hypercysteinemia does not have any effect on early ischemic stroke outcome [10] and in another study that has been done in Turkey in 2006 in order to determine the effect of serum homocysteine on acute ischemic stroke prognosis, it was demonstrated that although serum level of homocysteine was higher than normal in all days, it seems that there is not any association between serum level of homocysteine and acute ischemic stroke prognosis [12]. While in another study by Kado et al in 2002 it has been noted that there is an association between increased serum level of homocysteine and the risk of declined performance [19]. According to our precision in the selection of patients and matching them in the same location and extent of the lesion and the time of functional assessment, we can assert that our results have higher accuracy than their results. Of course, the only problem is that due to the lower number of hyperhomocysteinemic patients it could not be extensible.

One study by Pniewski in 2003 showed that the effect of homocysteine on 3 months prognosis of 74 patients was assessed more appropriately by Rankin score. Fourteen point two percent of patients with good Rankin score (0-2) and $66.7 \%$ of them with bad Rankin score (3-5) had mild hypercysteinemia and it was concluded that this factor increases the chance of bad rehabilitation 11.8 folds (95\% CI: 2.9 47.4) [20]. This investigation used also Rankin score and assessed the patients 3 months after stroke that can explain its difference with ours, maybe if the time of present study was longer, the same results were achieved and the negative effects of homocysteine were high-lightened.

In another study conducted in India from 2004 until 2006 by Kalita, severity of stroke based on Canadian Neurologic Severity measure and the patients' status were evaluated in 3 months after stroke with BI (good BI $>12$ and bad BI < 12). High serum level of homocysteine was seen among $60.6 \%$ of patients and low vitamin B12 and folic acid in $25.7 \%$ and $42.1 \%$ of patients respectively and finally it was concluded that patients with hypercysteinemia after 3 months had a better status [13]. In this study, for defining good and bad outcome, the cut-point of BI was determined as 12 wherever if a patient does all of asked activities with assistance, the minimum score obtained is 45 . So it seems that all of participated patients in worst status of function had good BI and so this cut-point is not an appropriate point. Therefore we have defined a new classification and divided to three categories including bad prognosis with BI lower than 40, moderate with BI 40 - 70 and good prognosis with $\mathrm{BI}$ higher than 70 . Although this categorization was more appropriate and logical, any significant correlation was found yet. Perhaps we could see a correlation if our consideration was the long-term outcome.

Homocysteine accelerates oxidative stress in ischemic stroke and plays a role in process of disease and affects function of patients and brain cells and if another compensatory mechanism does not exist, it can result in bad functional outcome. However, in our study homocysteine did not affect patients prognosis.

Evaluation of the homocysteine impact on mortality in the first week showed that after adjusting for confounding factors, it is a protective factor in front of mortality with OR equal to 1.179 , but this effect did not remain after 1 week till 1 month wherever some studies concluded that with its increasing the mortality rate increases $[2,21,22]$. Of course their findings do not violate our results, because their reports were not specified by special periods of time.

The interesting point of our study was the mean age of patients that deceased which is much less than alive patients in the first week $(58.4 \pm 15.2$ years and $70.0 \pm 11.7$ years $)$. In sum, patients who have died at the end of the first week were 12 years younger than alive patients but it reversed in the first month and average age of dead patient was more than alive patients $(70.82 \pm 13.91$ and $64.3 \pm 12.05$ years old $)$. This difference is significant. In other words, at the end of the first month, dead patients were 6 years older than alive patients, on average. According to cascade of cellular events, this can be explained, so in the first week, intra- and extra-cellular edema, size of the infarct area and brain herniation are the most common causes of death. Since in younger people the brain atrophy is less, herniation is more likely than older people. But after 1 week by reduction of cellular edema, the other factors such as the health of the other organs affect patients prognosis [3]. On the other hand, considering the protection effect of homocysteine against the first week mortality according to our results, and based on some studies that expressed that homocysteine level is lower in youngers than elders, the high rate of first week mortality in youngers may be the deprivation of this group from the protective effect of homocysteine. Also stroke in younger patients influenced by multiple factors that cause death in younger patients is higher than older patients in the first week after stroke.

\section{Conclusions}

Homocysteine decreases mortality rate in patients with stroke in the first week but does not affect the functional out- 
come of alive patients.

\section{Grant Support}

Guilan University of Medical Sciences, Vice Chancellorship of Research \& Technology.

\section{References}

1. Dharmarajan TS, Adiga GU, Norkus EP. Vitamin B12 deficiency. Recognizing subtle symptoms in older adults. Geriatrics. 2003;58(3):30-34, 37-38.

2. Naruszewicz M, Jankowska EA, Zymlinski R, Bukowska H, Millo B, Banasiak W, Ponikowski P. Hyperhomocysteinemia in patients with symptomatic chronic heart failure: prevalence and prognostic importance--pilot study. Atherosclerosis. 2007;194(2):408-414.

3. Biller J, Love BB, Schneck MJ. In: Bradley WG, Daroff RB, Fenichel GM, Jankovic J. Ischemiccerebrovascular disease. Neurology in clinical practice. Butterworth, Heinemann, 2008: 1165-1225.

4. Boudouresque J. [Introductory lecture on the clinical practice of neurology]. Arch Mediterr Med. 1966;43(6):295-329.

5. Kristensen B, Malm J, Nilsson TK, Hultdin J, Carlberg B, Dahlen G, Olsson T. Hyperhomocysteinemia and hypofibrinolysis in young adults with ischemic stroke. Stroke. 1999;30(5):974-980.

6. Boysen G, Brander T, Christensen H, Gideon R, Truelsen T. Homocysteine and risk of recurrent stroke. Stroke. 2003;34(5):1258-1261.

7. Linnebank M, Moskau S, Farmand S, Fliessbach K, Kolsch H, Bos M, Grothe C, et al. Homocysteine and carotid intima-media thickness in a german population: lack of clinical relevance. Stroke. 2006;37(11):28402842.

8. Verhoef P, Hennekens CH, Malinow MR, Kok FJ, Willett WC, Stampfer MJ. A prospective study of plasma homocyst(e)ine and risk of ischemic stroke. Stroke. 1994;25(10):1924-1930.

9. Barnett HMJ, Mohr TP, Stein BM, Yastu FM. Stroke: Pathophysiology Diagnosis and management. 3rd ed. New York:Churchill Livingston; 2000.

10. Okubadejo NU, Oladipo OO, Adeyomoye AA, Awosanya GO, Danesi MA. Exploratory study of plasma total homocysteine and its relationship to short-term outcome in acute ischaemic stroke in Nigerians. BMC Neurol. 2008;8:26.

11. Tagliari B, Zamin LL, Salbego CG, Netto CA, Wyse
ATS. Hyperhomocysteinemia increase damage on brain slices exposed to in vitro model of oxygen and glucose deprivation: prevention by folic acid. Int J Devl Neurosciences. 2006; 24:285-91.

12. Yoldas T, Gonen M, Godekmerdan A, Ilhan F, Bayram E. The serum high-sensitive $C$ reactive protein and homocysteine levels to evaluate the prognosis of acute ischemic stroke. Mediators Inflamm. 2007;2007:15929.

13. Kalita J, Kumar G, Bansal V, Misra UK. Relationship of homocysteine with other risk factors and outcome of ischemic stroke. Clin Neurol Neurosurg. 2009;111(4):364-367.

14. Mizrahi EH, Fleissig Y, Arad M, Adunsky A. Plasma homocysteine level and functional outcome of patients with ischemic stroke. Arch Phys Med Rehabil. 2005;86(1):60-63.

15. Kalita J, Srivastava R, Bansal V, Agarwal S, Misra UK. Methylenetetrahydrofolate reductase gene polymorphism in Indian stroke patients. Neurol India. 2006;54(3):260-263.

16. Panigrahi I, Chatterjee T, Biswas A, Behari M, Choudhry PV, Saxena R. Role of MTHFR C677T polymorphism in ischemic stroke. Neurol India. 2006;54(1):48-50; discussion 51-42.

17. Howard VJ, Sides EG, Newman GC, Cohen SN, Howard G, Malinow MR, Toole JF. Changes in plasma homocyst(e)ine in the acute phase after stroke. Stroke. 2002;33(2):473-478.

18. Khan U, Crossley C, Kalra L, Rudd A, Wolfe CD, Collinson P, Markus HS. Homocysteine and its relationship to stroke subtypes in a UK black population: the south London ethnicity and stroke study. Stroke. 2008;39(11):2943-2949.

19. Kado DM, Bucur A, Selhub J, Rowe JW, Seeman T. Homocysteine levels and decline in physical function: MacArthur Studies of Successful Aging. Am J Med. 2002;113(7):537-542.

20. Pniewski J, Chodakowska-Zebrowska M, Wozniak R, Stepien K, Stafiej A. Plasma homocysteine level and the course of ischemic stroke. Acta Neurobiol Exp (Wars). 2003;63(2):127-130.

21. Zhang W, Sun K, Chen J, Liao Y, Qin Q, Ma A, Wang $\mathrm{D}$, et al. High plasma homocysteine levels contribute to the risk of stroke recurrence and all-cause mortality in a large prospective stroke population. Clin Sci (Lond). 2010;118(3):187-194.

22. Bostom AG, Silbershatz H, Rosenberg IH, Selhub J, D'Agostino RB, Wolf PA, Jacques PF, et al. Nonfasting plasma total homocysteine levels and all-cause and cardiovascular disease mortality in elderly Framingham men and women. Arch Intern Med. 1999;159(10):10771080 . 